\title{
Establecimiento Penitenciario de Mediana Seguridad de Cartagena: una Nueva Oportunidad para la Salud
}

\author{
Medium Security Penitentiary Establishment of Cartagena: a New Opportunity for Health \\ ${ }^{a}$ Adriana Lucía Hernández Hernández ${ }^{65}$, María Isabel Ortega de Arco ${ }^{b}$, Daniela Ortega Pérez ${ }^{c}$, María Camila Puello Benitez ${ }^{d}$ \\ a adrihh98@gmail.com “Semillero Justicia, Política y Derecho”, Programa de Derecho, Fundación Universitaria Tecnológico Comfenalco. \\ Cartagena, Colombia. \\ b maryorte99@gmail.com “Semillero Justicia, Política y Derecho”, Programa de Derecho, Fundación Universitaria Tecnológico Comfenalco. \\ Cartagena, Colombia. \\ c dani0184@hotmail.com “Semillero Justicia, Política y Derecho”, Programa de Derecho, Fundación Universitaria Tecnológico Comfenalco. \\ Cartagena, Colombia. \\ dcmpbenitez@gmail.com “Semillero Justicia, Política y Derecho”, Programa de Derecho, Fundación Universitaria Tecnológico Comfenalco. \\ Cartagena, Colombia.
}

Forma de Citar: A.L. Hernández-Hernández, M.I. Ortega de Arco, D. Ortega Pérez et al., "Establecimiento Penitenciario de Mediana Seguridad de Cartagena; una Nueva Oportunidad para la Salud”, Rev. Saberes, Vol. 13, No. 02, pp. 93 - 98, 2020.

Recibido: 24/03/2020 Evaluación: 28/05/2020 Aceptado: 30/06/2020 DOI: https://doi.org/10.25213/1794-4384/1302.0013

\section{Resumen}

Desde hace varios años, la grave situación de salud dentro de las cárceles ha sido denominada como una problemática social, debido a que, contradice directamente los fines del estado, de bienestar e interés general, ocasionando fuertes críticas y reproches acerca de la función de la administración de velar por la vida digna de sus habitantes y eso no excluye a los que se encuentran en los centros penitenciarios. En consecuencia, esto, con sus diversas motivaciones, hizo de la defensa de los derechos humanos un tema político central en Colombia, para dar orden a estas problemáticas, el estado se manifestó creando legislación de protección e instituciones de defensa de los derechos humanos. La carta magna vigente, desde sus inicios proclama que Colombia es un estado social de derechos y brinda mecanismos para proteger los mismos, aunque estos se encuentren privados de la libertad no pierden su condición de persona y merecen los mismos derechos que los demás. Por ello, derechos como la vida, la salud y el acceso a la salud deben ser garantizados por el estado, que se presten eficazmente y no vulneren la vida digna que cada quien debe tener. En razón a lo anterior, éste trabajo busca mostrar cómo las

${ }^{65}$ Autor para correspondencia: correo electrónico adrihh98@gmail.com falencias del estado transgreden los derechos de los ciudadanos más frágiles: los reclusos.

\section{Palabras Claves}

Salud, dignidad humana, derechos fundamentales, vulneración, derechos, reclusos, constitución política.

\begin{abstract}
For several years now, the serious health situation in prisons has been described as a social problem, because it directly contradicts the aims of the State, the welfare and the general interest, causing strong criticism and reproaches about the role of the administration in ensuring the dignified life of its inhabitants, and that does not exclude those in prisons. Consequently, this, with its various motivations, made the defence of human rights a central political issue in Colombia. In order to solve these problems, the State demonstrated by creating legislation for the protection and defence of human rights. Since its inception, the Magna Carta proclaims that Colombia is a Social State of Rights and provides mechanisms to protect them, although they are deprived of their liberty, they do not lose their status as individuals and deserve the
\end{abstract}


same rights as others, for which reason rights such as life, health and access to health must be guaranteed by the State, that they are delivered effectively and do not violate the dignified life that everyone should have, so we want to show how the state's shortcomings violate the rights of the most fragile citizens, the prisoners.

\section{Keywords}

Health, human dignity, fundamental rights, infringement, rights, prisoners, political constitution.

\section{Introducción}

Las cárceles en Colombia, a lo largo de la historia han sido un sector caracterizado por la vulneración de derechos, tales como la salud, la dignidad humana, la vida, entre otros. Las condiciones de precariedad de los centros de reclusión agravan y transgreden las condiciones de vida digna que debe tener cada interno por su condición de persona. En consecuencia, a lo anteriormente planteado, en el presente proyecto se tiene por objetivo primordial identificar las falencias en la prestación del servicio de salud en el EPMSC de Cartagena en el año 2019 a partir del análisis de los informes proporcionados por entidades gubernamentales, análisis informativos, libros y tesis universitarias.

Este trabajo tiene por propósito identificar de forma específica, los planes político administrativos que implementa el distrito de Cartagena en el establecimiento penitenciario de Cartagena. Además, busca exponer las vulneraciones en el acceso al servicio de salud dentro del centro penitenciario de la ciudad. Por otro lado, busca impulsar la creación de una fundación, la cual, se encargará de prestar asesorías legales a los reclusos que consideren que hay una vulneración por parte del Distrito de Cartagena en la prestación del servicio de salud, debido a que las condiciones de precariedad y la administración del centro de reclusión de Cartagena, transgrede el derecho a la salud, sabiendo que este es un derecho de carácter constitucional. Finalmente busca responder pregunta problema ¿Cómo se vulnera el acceso al derecho fundamental a la salud en el establecimiento penitenciario de mediana seguridad y carcelario de Cartagena en el 2019?

\section{Metodología}

La metodología empleada para realizar este artículo tiene un enfoque cualitativo y documental, entendiéndose por este, aquel enfoque de las investigaciones que tienen como objeto principal realizar descripciones detalladas de situaciones, eventos, personas, interacciones $\mathrm{y}$ comportamientos que son observables. (Acosta, J., Ferrer, N., Villegas, D. 2018 p 52). Así mismo con este enfoque tiene un carácter socio jurídico, la cual se encuentras totalmente centrada en el funcionamiento real de las normas jurídicas, abarcando el aspecto social, no solo desde la sociología sino desde cualquier disciplina (Acosta, J., Ferrer, N., Villegas, D. 2018 p 58, 59 y 60).

De igual manera, se realizó un rastreo bibliográfico de manera detallada, para así obtener las bases teóricas del tema a trabajar que ya ha sido anteriormente mencionado. Así mismo, para poder determinar que este establecimiento penitenciario vulnera el acceso al derecho a la salud de sus reclusos y no se encuentra en sus mejores condiciones, se tuvo como punto de referencia informes proporcionados por la procuraduría regional y distrital, la defensoría del pueblo y numerosos reportes periodísticos.

\section{Planes político administrativos que implementa el Distrito de Cartagena en el Establecimiento Penitenciario de Cartagena.}

Para el desarrollo del buen funcionamiento de la administración, se han estructurado unas políticas administrativas entre el Distrito y el INPEC. Gracias a un estudio realizado por la Defensoría del Pueblo acerca de las deficiencias que existen en la alimentación en la cárcel de Cartagena, se logró evidenciar serias carencias en la calidad de los alimentos, el valor nutricional, su estado de conservación y almacenamiento, lo que se traduce en la entrega de alimentos en condiciones contrarias a las recomendaciones nutricionales de las autoridades sanitarias, lo cual constituye un atentado a la dignidad humana de estos reclusos. En una visita realizada por esta misma entidad, se pudo comprobar que la Unión Temporal Alianza para el Futuro 2011, delegada de la alimentación

Página | 94 
de los internos, mantenía en pésimas condiciones higiénicas y sanitarias el procesamiento y suministro de las raciones de comida. Luego de recibir las quejas de los internos, Otálora Gómez le solicitó a la Contralora General de la República, Sandra Morelli, la revisión del contrato de alimentación de dicho establecimiento penitenciario y carcelario debido al elevado número de quejas de los reclusos recogidas durante la visita de inspección. Tras esta solicitud, la Contraloría General de la República estableció un presunto detrimento patrimonial por valor de $\$ 128$ millones por irregularidades en la ejecución del contrato de suministro de alimentos para los internos de la cárcel de Ternera de Cartagena, exactamente por ración facturada y no suministrada. (Defensoría del Pueblo, 2014, p. 40).

Para el año 2019 el distrito de Cartagena firmó un convenio interadministrativo por el valor de 600 millones con el INPEC, para destinar el servicio y la remuneración que establece la Ley 65 de 1993 en caso de que un departamento o municipio carezca de cárcel propia para hacerse cargo de las personas indiciadas o sindicadas con detención preventiva; los cuales según un informe periodístico realizado por el periódico el Universal, serían distribuidos así: el $30 \%$, es decir, 180 millones de pesos, para el pago del sobresueldo de los empleados del establecimiento penitenciario; y $70 \%$, correspondiente al valor de 420 millones de pesos, para el suministro de elementos para la atención, rehabilitación y tratamiento de los internos.

Se observa que el distrito de Cartagena debió adquirir tales bienes, iniciando los trámites contractuales, y entregarlos al INPEC, es decir, no se reciben recursos presupuestales, sino que se adquieren obligaciones cuya ejecución se materializará en actos jurídicos posteriores que comprometen presupuestalmente al Distrito de Cartagena.

Lo anterior quiere decir que los encargados de adquirir dichos bienes son los funcionarios que el distrito disponga para ello, pero ¿Qué tan optimo es que estos funcionarios sean quienes dispongan del dinero?, sabiendo que son personas que desconocen las auténticas necesidades de los internos; que no disponen de la conciencia de la realidad del centro penitenciario; la lógica colectiva podría llegar a inferir que lo más óptimo para esto sea hacer entrega directa de los activos a los funcionarios del INPEC para que sean ellos quienes dispongan de cuáles serán los destinos de estos recursos.

\section{Vulneración al derecho a la salud dentro del} centro penitenciario y carcelario de mediana seguridad en Cartagena en el año 2019.

Las cárceles son instituciones que reciben personas que provienen de todos los estratos sociales, por lo que pueden ser consideradas como una población que refleja la estructura y dinámica de la sociedad, es por esto que, como estructura social deben contar con un sistema político administrativo acorde a sus necesidades. Las condiciones de precariedad de los centros de reclusión agravan y trasgreden las condiciones de vida digna que debe tener cada interno, estas vulneraciones han generado preocupación en diversos órganos independientes del estado, como lo son la Contraloría Delegada para la Participación Ciudadana, la cual, realizo a una investigación y mediante escrito planteo que:

"El Centro Penitenciario y Carcelario de Ternera no cuenta con unas instalaciones con capacidad adecuada para la elaboración de los alimentos de la población carcelaria, teniendo en cuenta que su capacidad es para 1.000 internos y en la actualidad se encuentran alrededor de 2.200 internos. Además, no reúne las condiciones mínimas higiénicas y sanitarias requeridas en el mismo Contrato 031 de 2011 adjudicado a la Unión Temporal Alianza para el Futuro, que en 2011 prestaba sus servicios a 16 centros penitenciarios a nivel nacional". (Defensoría del Pueblo, 2014 p. 41).

La precariedad alimenticia, es prueba fehaciente de que los reclusos del centro carcelario no habitan o conviven en óptimas condiciones. El estado ha sido amonestado para regular ese tipo de situaciones y no hagan pie para una situación de Estado Inconstitucional.

De acuerdo al planteamiento realizado en la sentencia T-153 de 1998, se establece que las cárceles en Colombia se encuentran en Estado de cosas inconstitucional y que su estructura transgrede lo establecido en el ordenamiento jurídico colombiano. Las cárceles colombianas se caracterizan por el hacinamiento, las graves deficiencias en materia de servicios públicos y

Página | 95 
asistenciales, el imperio de la violencia, la extorsión y la corrupción, y la carencia de oportunidades y medios para la resocialización de los reclusos.

Esta situación se ajusta plenamente a la definición del estado de cosas inconstitucional, de allí se deduce una flagrante violación de un abanico de derechos fundamentales de los internos en los centros penitenciarios colombianos, tales como la dignidad, la vida e integridad personal, los derechos a la familia, a la salud, al trabajo y a la presunción de inocencia, etc. En realidad, el problema carcelario representa no sólo un delicado asunto de orden público, como se percibe actualmente, sino una situación de extrema gravedad social que no puede dejarse desatendida y que las circunstancias en las que transcurre la vida en las cárceles exigen una pronta solución. Pero el remedio de los males que azotan al sistema penitenciario no está únicamente en las manos del INPEC o del Ministerio de Justicia. Por eso, la corte tiene que pasar a requerir a distintas ramas y órganos del Poder Público para que tomen las medidas adecuadas en dirección a la solución de este problema. (Sentencia, T-153 de 1998).

Por otra parte, en un acta obtenida de la Procuraduría General de la Nación, emitida el 31 de mayo del 2018, se logró confirmar una vez más la falta diligencia que existe hacia el centro penitenciario y carcelario de mediana seguridad en Cartagena, donde el principal tema abordado fue la salud y la prestación de servicios asistenciales de urgencias y remisiones de internos a los distintos puntos de atención externos en salud. Se expone, por parte de los miembros del comité reunido (Procuradores Judiciales Penales, jueces de Ejecución de Penas y Medidas de seguridad, comité de Derechos Humanos, director del INPEC, entre otros.) la falta de medicamentos, aseverando que se trata de una situación que permanece en el tiempo, indicando la falencia que existe para el suministro de los medicamentos requeridos por la población interna. Refiere que ser internos no debe perjudicar sus derechos fundamentales de acceso a la prestación de servicios médicos en los distintos niveles de atención. Sobre el planteamiento de este problema interviene el señor director del INPEC, que se han optado medidas orientadas a hacerle frente a la situación que se denota, procurando con ello mejorar las condiciones de atención médica a la población carcelaria, dando cuenta que la Fiduprevisora contrata a 'Genéricos Esenciales' para asegurar un mejor estándar en los suministros de medicamentos requeridos.

El director también pone en conocimiento que hay un solo médico disponible 24 horas todos los días, los sábados hasta mediodía y los domingos no hay médico disponible.

La 'Cárcel de Ternera' como coloquialmente es conocida, debería albergar solo a 1.464 internos condenados, que es su verdadera responsabilidad, pero en gráficas proporcionadas por la procuraduría distrital de Cartagena, se pudo observar que hoy día hospeda a 2.320, donde más de mil son los sindicados, contribuyendo a que el estado de precariedad, hacinamiento y por lo tanto la salud de la cárcel se haga aún más grave.

Tras la liquidación de CAPRECOM y el ingreso de 'Genéricos esenciales' como nuevo encargado de prestar el servicio de salud y suministrar los medicamentos, la situación en el EPMSC no ha mejorado, pues siguen presentándose múltiples falencias en cuestión de salud, que día a día van haciendo menos llevadera la situación para quienes están pagando una pena privativa de la libertad.

Dentro de este establecimiento penitenciario se han venido adelantando campañas de salud y programas de prevención de enfermedades por parte del Distrito; esto con el fin de mejorar la calidad de vida de los internos y prevenir la aparición de nuevos brotes de enfermedades. En abril del presente año se iniciaron cuatro programas de prevención de enfermedades y una campaña de vacunación, a pesar de que la iniciativa es compasiva y aceptable ya que eventualmente cumple con la función del Estado de garantizarle a estas personas el derecho a la salud, han sido insuficientes, pues es poco para los 126 enfermos que tenía el plantel carcelario para la fecha. Dentro de la cárcel se pueden encontrar enfermedades como VIH, cáncer, tuberculosis, entre otras, sin contar aquellas enfermedades de las cuales los otros reclusos podrían contagiarse por la constante convivencia con aquellos que ya están enfermos; sumándole a esto la falta de personal para prestar servicios óptimos de salud, la deficiencia en el proporcionamiento de medicamentos y la falta de posibilidades para el traslado de los reclusos a un lugar donde sean 
atendidos de forma correcta. El incremento de enfermedades también se le puede atribuir a las condiciones insalubres en las cuales se encuentran los reclusos, que van desde aguas servidas o residuales, hasta la convivencia con roedores.

Las ya mencionadas medidas de prevención y atención de enfermos deben ser sometidas a una reevaluación sobre su eficacia, pues el abandono que se presenta por parte del Estado a estas personas permite que se infiera la poca importancia que generan para los altos mandatarios, tal parece que estar privado de la libertad por pagar una condena es sinónimo de ser invisible para una nación cuyo principal fin debe ser siempre favorecer a aquellos que no tienen un medio para obtener sus propios beneficios.

En cuanto a las obras para las mejoras de la cárcel, en el acta adquirida de la Procuraduría General de la nación, se habla de la existencia de una contratación administrativa entre el USPEC y FONADE, por la suma de $\$ 13.685 .891 .840$ de pesos para arreglos locativos que contribuyan a la reparación general de "el Rancho", donde se preparan los alimentos del penal, y obras en las garitas de vigilancia, pavimentación de muros y construcción de alojamientos de guardias (entre otras). De Ávila, (2018) afirma. "la cocina transitoria se nos está quedando fija, tampoco cumple con estándares, no se ha iniciado las obras en "El Rancho". Pedimos al DADIS que cierre el área de producción de alimentos de la Cárcel de Ternera". (p. 1)

Hasta la fecha, por un informe publicado por $E l$ Universal, se pudo conocer declaraciones del sindicato del INPEC, donde se establece que al parecer las obras no han empezado.

Recapitulando lo anterior mencionado, se puede constatar que existen diversas causales que afectan el desarrollo del derecho humano fundamental a la salud, entre las cuales encontramos la política criminal desarticulada, que ha optado por un "endurecimiento punitivo", efectuándose con el aumento de penas mínimas para algunos delitos; por otro lado el hacinamiento carcelario, hace que sea desproporcional el número de reclusos y la capacidad de cupos de los establecimientos penitenciarios, produciendo esto condiciones insalubres.

Además, la reclusión conjunta de sindicados y condenados fortalece esta vulneración, la Corte había emitido órdenes con el objetivo de eliminar la reclusión unida de éstos. Sin embargo, la problemática sigue vigente $\mathrm{y}$, según la corte, se debe principalmente a dos aspectos: primeramente, reformas legislativas que han hecho más rigurosa y obligatoria la implementación de medidas de aseguramiento privativas de la libertad; en segundo lugar, otorgar un tratamiento diferenciado a las personas sindicadas y condenadas.

El ineficaz sistema de salud no da abasto para toda la población recluida, prueba de ello, es que hasta la fecha algunos internos siguen solicitando tratamientos médicos por vía tutela. Las cárceles continúan desproveídas de medicamentos y otros insumos, situación denunciada por el sindicato del INPEC y confirmado por la Defensoría del Pueblo. A esto se suma la dificultad para la asignación de citas, la falta de cuidado de especialistas y atención especial para adultos mayores o madres gestantes, entre otros.

En definitiva, Colombia aun siendo un estado social de derecho con tantas garantías constitucionales y ratificación de tratados internacionales, aún realiza estás transgresiones, a derechos de primer orden, lo que realmente es incoherente e inconstitucional, dado que, va en contra a lo establecido en la carta magna, a pesar de que, el estado es quien tiene la obligación de velar por sus habitantes, es el mismo quien no los protege.

\section{Fundación REDES: Reclusos por el Derecho a la Salud.}

La Fundación REDES nace como un mecanismo de solución a las situaciones actuales donde se les ha vulnerado el derecho a la salud a los reclusos del centro penitenciario y carcelario de mediana seguridad de Cartagena; el objetivo de esta fundación es brindar acompañamiento jurídico y asesorías gratuitas a aquellos reclusos que consideren que su derecho a la salud está siendo vulnerado por parte del distrito de Cartagena y, por ende, del estado colombiano. La razón lleva a la creación de esta fundación es principalmente la precariedad del servicio a la salud del centro penitenciario y la afectación que tienen los reclusos como una consecuencia de ello; sin embargo, también se puede constatar un déficit a nivel de conocimiento de sus derechos y las acciones constitucionales que tienen para

Página | 97 
defenderlos y solicitar que sean cumplidos, esto conlleva a que al desconocer de sus derechos y de los mecanismos para exigir el cumplimiento de estos los hagan más vulnerables a la violación por parte del estado; siendo esta otra razón encontrada para que se presente la necesidad de esta fundación como mecanismo que ayude a exigir la protección y el cumplimiento del derecho a la salud.

Esta Fundación busca, no solo solucionar los problemas que se presenten de transgresión al derecho a la salud, sino también generar espacios de dialogo con los órganos estatales como defensoría del pueblo, directores de centros penitenciarios y demás entes capacitados para brindar este servicio y obtener una mejoría significativa que permita que los internos de estas penitenciarias obtengan su derecho a la salud de forma óptima e ininterrumpida.

\section{Conclusiones}

Se lograron identificar diferentes causales que afectan el acceso al derecho fundamental a la salud en el EPMSC de Cartagena, tales como; los planes político administrativos que implementa el Estado deben ser vigilados e inspeccionados por un órgano independiente, las vulneraciones dentro del EPMSC de Cartagena se originan por el hacinamiento, la carencia de elementos quirúrgicos y medicación para contrarrestar las enfermedades. Colombia, aun siendo un Estado Social de Derecho con tantas garantías constitucionales y ratificación de tratados internacionales, realiza estas transgresiones a derechos de primer orden, lo que realmente es incoherente e inconstitucional.

\section{Referencias Bibliográficas}

Acosta, J., Ferrer, N. y Villegas D. (2018) "Metodología de la investigación jurídica y sociojurídica”. Cartagena, Colombia p 52, 58, 59, 60 Ed. Bonaventuriana.

Alto Comisionado de las Naciones Unidas para los Derechos Humanos, (2001). Misión internacional, derechos humanos y situación carcelaria, p. 8 .
Bobbio, N., (2003) "Teoría general de la Política". Madrid, España. Ed.2 Trotta, p 520.

De Ávila, J. (21 de mayo de 2018). Cárcel San Sebastián de Ternera no aguanta más. El Universal. Recuperado de http://www.eluniversal.com.co/cartagena/carcelsan-sebastian-de-ternera-no-aguanta-mas-278848JBEU394580

Meza, M. (16 de marzo de 2019). INPEC y Distrito firman convenio por $\$ 600$ millones. El Universal. Recuperado de https://www.eluniversal.com.co/cartagena/inpecy-distrito-firman-convenio-por-600-millones$\underline{\mathrm{DY} 908050}$

Defensoría del Pueblo. (2014). Vigésimo primer informe del Defensor del Pueblo al Congreso de la República. Bogotá: Defensoría del pueblo, p. 40,41 .

Constitución política de Colombia [Const.] (1991) Artículo 2 [De los principios fundamentales]. 2da Ed. Legis. 